\title{
Abstracts - AGE Sesssion in Berlin (October 18, 2013)
}

Is radical surgery always appropriate for the treatment of deep infiltrating endometriosis?

\section{U. Ulrich}

Department of Obstetrics and Gynecology, Martin Luther Hospital, Berlin, Germany

The treatment of choice for DIE - in general - is complete resection which often means: radical resection. However, radical resection has the potential of serious complications. Some reflections may be allowed: First, how often endometriosis might be a finding rather than a disease? As long as there is no pain, no destruction of an organ, and no infertility, there may be no indication of surgery. Also, with respect to the disease per se, complete resection is the best treatment. However, we are usually dealing with young women with the need to have their fertility preserved - so incomplete surgery often is the necessary result of the operative intervention. When, for example, is rectal resection advocated? Perhaps, if the present situation of the patient is worse than potential complications of the operation, you have got a fine indication. Another consideration may be helpful when a decision as to an operation has to be made in women with endometriosis-associated infertility: Would you as the surgeon operate on the patient if she had, say, five children? In older women, one should always be on the watch if endometriosis-associated symptoms become worse after the menopause as this may be the only clinical sign of a malignant transformation of the endometriosis that the patient is known to have suffered from previously. In conclusion: Not every patient with deep infiltrating endometriosis has to undergo radical resection. It is the proper indication - i.e., pain, organ destruction, and infertility - that counts. We should operate on patients with endometriosis as infrequent as possible, and we should only operate on patients, not on findings.

\section{Performing bowel anastomosis - feasible also for Gynaecologist?}

\section{A. Hackethal, Brisbane, Australia}

There is a good amount of literature reflecting the need for surgical management of bowel disease with anastomosis in the speciality of Gynaecology. A literature review from 1986 to 2013 with the search terms „bowel and rectal anastomosis in gynaecology“ identified 147 publications, whereby 29 where relevant. The vast majority deals with surgery in gynaecological oncology and endometriosis, however, some are highlighting necessary training curricula for performance of bowel surgery.

Independent from the kind of specialist doctor performing bowel surgery, considerations should be given to primary patient factors. Bowel surgery can and should be considered in oncologic patients who can be optimally debulked and those, who will have reduced or delayed obstruction symptoms. In endometriosis, radical surgery is known to play an important role in pain reduction. However, extensive surgery is associated with increased morbidity and therefore mortality and should only be performed in trained and adequately equipped units.
Patient factors influencing bowel surgery comprise patient medical history, co-morbidities and Albumin levels as example. Surgical wise, the location and number of bowel anastomosis influence the postsurgical outcome. In general, bowel anastomosis should have a good blood supply, no tissue tension and no distal obstruction. Additionally, considerations for a protective ileostomy should be given.

Though, technically bowel surgery can be straight forward and with the use of stapling devices reasonably fast, the importance for safe and effective surgery is based on an interdisciplinary approach. Gynaecologists who do not have a formal general surgical training and are not regularly performing bowel surgery should involve a general surgical team for these procedures.

Hysterectomy trends over a nine-year period: an endoscopic teaching-center experience

Thoralf Schollmeyer; Mohamed Elessawy; Babis Chastamouratidhs; Ibrahim Alkatout; Ivo Meinhold-Heerlein; Liselotte Mettler; Walter Jonat; Marion T Weigel

Department of Obstetrics and Gynaecology, University Hospital, Kiel, Germany

Objective: This study aimed to investigate the development in the performance of hysterectomies in a single certified endoscopic teaching center.

Methods: Data was collected retrospectively from 954 patients who underwent hysterectomy between 2002 and 2010 for benign indications at the Department of Obstetrics and Gynecology, UKSH, Campus Kiel, Germany.

Results: The most frequent indications for hysterectomy were uterine myoma, adenomyosis, prolapse, hyperplasia of uterus and cervix, menstrual disorder and endometriosis. The shortest operating time was recorded for $\mathrm{VH}$ and the longest for LAVH. The average uterine weight was the highest for $\mathrm{AH}$ and lowest for $\mathrm{VH}$. The lowest complication rate was recorded for LASH $(2.4 \%)$ and the highest for AH $(6.6 \%)$. The two laparoscopic techniques for hysterectomy (LASH and TLH) showed a lower complication rate than $\mathrm{VH}$ and $\mathrm{AH}$. The highest intraoperative complication rate occurred at abdominal hysterectomy (4.5\%) and the lowest at TLH (1.5\%). The major postoperative complication rate was $1.8 \%$. The highest incidence of major complications was recorded for LAVH (6.4\%).

Conclusion: Within the last years laparoscopic operations have increased significantly and are associated with lower complication rates. Laparoscopic hysterectomies (LASH, TLH) lead to a decrease in the complication rate despite the higher risk score of these patients.

\section{Laparoscopic surgery in patient with ovarian cancer}

\section{K.J. Neis}

Department of Obstetrics and Gynaecology, Homburg, University Medical Centre, Homburg, Germany 
The guideline "Laparoscopic operations of ovarian tumors" was published in the first edition 15 years ago. The aim was to avoid laparoscopic surgery in patients with ovarian cancer.

Today laparoscopic surgery is also a standard procedure in oncology especially in cervical- and endometrial cancer. So it is time to reflect whether there is also an indication for laparoscopic surgery in ovarian cancer. In the literature we find a small number of publications about minimal invasive surgery in early ovarian cancer (EOC). It seems that there is no difference in the clinical outcome between laparoscopic surgery and laparotomy. For minimal invasive surgery no new technique must be developed: adnexectomy, resection of peritoneum, pelvic-and paraaortal lyphonodectomy are well established in laparoscopy.

This means the laparoscopic surgery for EOC is in accordance to the Principe of Good Medical Practice when performed in an individual case. To establish the method as a routine procedure a multi center study should be initiated. This study should help to develop best option regarding all oncological and surgical aspects in this field.

\section{Significance of sentinel node biopsy in patients with endometrial- and cervical cancer}

E.-F. Solomayer, Department of Obstetrics and Gynaecology, Homburg University Medical Centre, Homburg, Germany

\section{Abstract}

In the last two decades, sentinel lymph node biopsy has completely changed the proceeding of surgical oncology. In the beginning, patients with small primary tumors of the breast or skin with regional lymph nodes no longer suffer the consequences of regional lymphadenectomy, in particular lymphedema. During this period, numerous studies of the sentinel lymph node biopsy technique in patients with gynecological cancers, including endometrial and cervical cancer have been performed. From a technical point of view, using the combined technique of blue dye and radiocolloid will yield the best results using Technetium 99 (Tc 99) as well as acid blue as tracer in order to assign the sentinel node(s) in the pelvis. Sentinel detection is thereby possible doing laparoscopy or laparotomy, independently from the surgical access. Concerning endometrial cancer, the available data about sentinel node biopsy is very inconsistent, and up to date there are no prospective studies. Therefore, currently sentinel node biopsy for endometrial cancer should still be considered in the feasibility stage of development. In contrast, in early cervical cancer several studies showed auspicious results for patients with small tumors $(<2 \mathrm{~cm})$. However, in patients with locally advanced cervical cancer, sentinel node biopsy is disaffected since it is associated with high false negative rates as low sentinel detection rates. Summing up, sentinelnode biopsy in gynecogical cancer might be a promising technique in early stage cervical cancer though has to be considered cautious in endometrial cancer due to the insufficiency of the available data. 